\title{
¿En qué confiamos al evaluar la calidad de las universidades? Evolución de los mecanismos externos de aseguramiento de la calidad en Chile
}

Julio Labraña y César Mariñez

\section{RESUMEN}

Los sistemas contemporáneos de educación superior dependen de la existencia de instituciones responsables de asegurar la calidad de sus actividades. El caso chileno no es una excepción en este sentido. Con la creación del Consejo de Educación Superior en 1990 comienza en este país el desarrollo de un sistema de evaluación experta de las universidades, consolidado con la apertura de la Comisión Nacional de Acreditación en 2006. A pesar de su importancia, no parecen existir estudios acerca de la historia de estos mecanismos de aseguramiento de la calidad, ni de cómo su evolución puede explicarse desde una teoría sociológica general. Para avanzar en este análisis, siguiendo la tesis de Niklas Luhmann, se argumentará que la confianza es socialmente construida y que su forma de configuración depende de cambios en la estructura de la sociedad. Utilizando estas ideas, se examina la evolución de los mecanismos de aseguramiento de la calidad de la educación superior desde el siglo XIX en Chile, sugiriendo que el énfasis en el conocimiento experto es un fenómeno reciente que puede ser explicado como consecuencia del cambio en el modo de estructuración de la sociedad. Este análisis sociológico, desde la teoría de sistemas sociales de Luhmann, finaliza con un resumen, posibles líneas de investigación y recomendaciones de política de educación superior.

Palabras clave: sistemas de aseguramiento de la calidad, confianza institucional, educación superior chilena, sistemas expertos, Chile.

Juli Labraña

jlabrana@ug.uchile.cl Chileno. Doctor por la Universität Witten/Herdecke, Alemania. Investigador del Centro de Políticas Comparadas de Educación, Universidad Diego Portales, Chile. Núcleo de Estudios Sistémicos Transdisciplinarios. Temas de investigación: educación superior, teoría sociológica y estudios en el vínculo entre semántica y estructura.

\section{César Mariñez}

cdmarinez@uchile.cl Chileno. Doctor en Filosofía, mención Moral y Política, Universidad de Chile. Investigador en el Núcleo de Innovaciones Efectivas en Política Pública (Nıepp), Departamento de Trabajo Social, Universidad de Chile. Temas de investigación: ética social, confianza organizacional e institucional. 


\title{
Em que confiamos ao avaliar a qualidade das universidades? Evolução dos mecanismos
} externos de garantia da qualidade no Chile

\section{RESUMO}

Os sistemas contemporâneos de educação superior dependem da existência de instituições responsáveis de garantir a qualidade de suas atividades. O caso chileno não é uma exceção neste sentido. Com a criação do Conselho de Educação Superior em 1990 começa neste país o desenvolvimento de um sistema de avaliação experta das universidades, consolidado com a introdução da "Comisión Nacional de Acreditación" em 2006. Apesar de sua importância, não parecem existir estudos sobre a história destes mecanismos de garantia da qualidade, nem de como sua evolução pode ser explicada desde uma teoria sociológica geral. Para avançar nesta análise, seguindo a tese de Niklas Luhmann, se argumentará que a confiança é socialmente construída e que sua forma de configuração depende de mudanças na estrutura da sociedade. Utilizando estas ideias, se examina a evolução dos mecanismos de garantia da qualidade da educação superior desde o século XIX no Chile, sugerindo que a ênfases no conhecimento experto é um fenômeno recente que pode ser explicado como consequência da mudança no modo de estruturação da sociedade. Esta análise sociológica, a partir da teoria de sistemas sociais de Luhmann, finaliza com um resumo, possíveis linhas de pesquisa e recomendações de política de educação superior.

Palavras chave: sistemas de garantia da qualidade, confiança institucional, educação superior chilena, sistemas expertos, Chile.

\section{What do we rely on when evaluating the quality of universities? Evolution of external quality} assurance mechanisms in Chile

\begin{abstract}
The contemporary higher education systems depend on the existence of institutions responsible for ensuring the quality of their activities. The Chilean case is no exception in this regard. The creation of the Higher Education Council in 1990 was the starting point for the development of an expert assessment system for universities, consolidated with the inauguration of the National Accreditation Commission in 2006. Despite their importance, there do not seem to be studies on the history of these quality assurance mechanisms, nor on how their evolution can be explained from a general sociological theory. To make progress in this analysis, the authors, following Niklas Luhmann's thesis, put forward that trust is socially constructed and that its form of configuration depends on changes in the structure of society. Using these ideas, the evolution of quality assurance mechanisms in higher education since the 19th century in Chile is reviewed and the results suggest that the emphasis on expert knowledge is a recent phenomenon which can be explained as a consequence of the change in the way society is structured. This sociological analysis, based on Luhmann's theory of social systems, ends with a summary, possible lines of research, and recommendations for higher education policy.
\end{abstract}

Key words: quality assurance systems, institutional trust, chilean higher education, expert systems, Chile.

Recepción: 03/07/18. Aprobación: 28/05/19 


\section{Introducción}

La educación superior se ha convertido en las últimas décadas en una de las variables fundamentales del desarrollo económico de los países (World Bank, 2002; Luke, 2006; Rodríguez-Ponce, 2009; Välimaa y Hoffman, 2008; Kim, 2014). No debe sorprender, por lo tanto, el aumento de interés de gobiernos, así como de los administradores y estudiantes de estas instituciones, por la determinación de la calidad de los servicios educacionales que ellas ofrecen. Una de las muestras más evidentes de este interés en la calidad de la formación universitaria es la creación en las últimas décadas de sistemas expertos externos dedicados a la evaluación de estas instituciones, los así llamados sistemas de aseguramiento de la calidad de la educación superior. Generalmente, estos organismos establecen regulaciones para asegurar la transparencia de las universidades y definen, además, estándares para su correcto desempeño.

Una vez constituidos como tales, estos organismos tienden a operar de manera relativamente autónoma respecto de los gobiernos, al menos en lo que respecta a la aplicación de sus criterios. Como resume Neave (1998), su objetivo es institucionalizar una forma de regulación de los sistemas de educación superior que, en lugar de controlar directamente las actividades de las instituciones o dejarlas liberadas a su evolución independiente, sea transparente en sus características esenciales y promueva la racionalidad en el proceso de toma de decisiones de los distintos actores interesados en su evolución y desarrollo.

El caso de Chile no parece ser una excepción en esta tendencia. En 1990, la Ley Orgánica Constitucional de Enseñanza estableció la creación del Consejo Superior de Educación (CSE), conformado por representantes del gobierno y de las universidades estatales y privadas, institutos profesionales, academias del instituto de Chile, la Corte Suprema de Justicia, el Consejo Superior de Ciencias y las Fuerzas Armadas (CSE, 1991). En particular, la función asignada a este órgano fue pronunciarse acerca de los proyectos institucionales presentados por universidades e institutos profesionales para tener el reconocimiento por parte del Estado y, posteriormente, guiarlos hasta que pudiesen operar autónomamente.

A continuación, tras un extenso debate legislativo, en 2006 se aprobó la creación de la Comisión Nacional de Acreditación (CNA). Este órgano está conformado por representantes del gobierno y de los institutos profesionales, de las universidades pertenecientes al Consejo de Rectores de las Universidades Chilenas (CRUCH), de las universidades privadas, de la Comisión Nacional de Investigación Científica y Tecnológica (CONICyT), del sector productivo, de asociaciones disciplinarias o profesionales del país y de los estudiantes. Sus principales funciones son acreditar las instituciones de educación superior autónomas, pronunciarse acerca de las solicitudes de autorización presentadas por agencias privadas, encargadas de evaluar programas de pregrado y, por último, supervigilar su adecuado funcionamiento.

La centralidad de estas organizaciones en el aseguramiento de la calidad de la formación ofrecida por las instituciones de educación superior chilenas no encuentra antecedentes directos. Sin embargo, a pesar de su importancia actual en términos de política pública y su carácter inédito en términos históricos, pareciese que su relación con los cambios en el fenómeno de la confianza no ha sido aún analizada sociológicamente. Por el contrario, la mayor parte de aproximaciones en torno a este sistema de aseguramiento de la calidad se enfoca en la caracterización de su evolución reciente (Lemaitre, 2004; Lemaitre y Zenteno, 2016), sin integrarlos en el marco de una hipótesis teóricaestructural (para un esfuerzo en esta dirección, véase Salazar y Leihy, 2013). Como se verá en este artículo, esto dificulta la comprensión del significado histórico de estos sistemas de evaluación de las universidades e impide observar su dependencia respecto a desarrollos estructurales característicos de la sociedad moderna.

Considerado lo anterior, resulta interesante para la investigación sociológica observar cuáles son las 
condiciones de posibilidad relativas a la confianza en que el actual sistema de evaluación ha adquirido importancia. Al respecto, en este estudio se argumentará que estos sistemas expertos dependen de la existencia de confianza acerca de la validez de sus procedimientos para desempeñar su rol esperado pues, caso contrario, sus decisiones no serían consideradas como relevantes por otros actores interesados.

La dependencia de estos sistemas respecto a la confianza convierte el estudio de su evolución en una materia extremadamente relevante tanto para los estudios en educación superior como para las políticas públicas. Las variaciones en las formas de confianza, como se sostendrá en este artículo, no pueden comprenderse fuera de cambios sociales (Luhmann y Schorr, 2000). Con ello, la comprensión sociológica del significado de los sistemas de aseguramiento de la calidad actuales implica necesariamente el análisis de la evolución de formas anteriores usadas para asegurar la calidad de las universidades, formas en las que, como hoy, se confiaba en su capacidad de regulación del sistema.

Este es el objetivo del presente artículo. Para probar esta afirmación, el artículo se estructura en las siguientes secciones. En primer lugar, se describe la importancia de los mecanismos de aseguramiento de la calidad. A continuación, se sugiere que estos mecanismos no pueden entenderse fuera de un análisis del rol de la confianza en la sociedad moderna y se arguye sobre la conveniencia de utilizar la teoría de sistemas sociales desarrollada por Niklas Luhmann como marco para comprender estos cambios. Siguiendo este diagnóstico, la tercera sección propone la hipótesis interpretativa que guía este estudio. Enunciada sucintamente, ella es que la evolución de los mecanismos de aseguramiento de la calidad de la educación superior chilena es expresión de transformaciones estructurales en la forma primaria de confianza en la sociedad contemporánea, que responden al tránsito desde una comprensión de la confianza basada en la familiaridad, a otra basada en técnicas de evaluación expertas. La cuarta sección utiliza esta hipótesis para examinar la evolución de los mecanismos de aseguramiento de la calidad de las actividades de las universidades chilenas. El artículo finaliza con un breve resumen, posibles líneas futuras de investigación y recomendaciones de política pública.

\section{Sistemas de aseguramiento de la calidad y educación superior}

La emergencia de mecanismos de aseguramiento de la calidad de las instituciones de educación superior es consecuencia de diversos factores inmediatos. Entre los más importantes, está el cambio en la tradicional forma de relación entre gobierno y universidades como consecuencia de la crisis del Estado de Bienestar. De esta manera, en lugar de transferir recursos no sujetos a resultados, como era característico en las distintas versiones del Estado benefactor, los gobiernos tienden hoy en día a evaluar cuáles son los efectos específicos de las asignaciones al sector (Vidovich y Currie, 2011). Al mismo tiempo, existe un proceso de diversificación de los sistemas nacionales de educación superior, tanto en términos de las funciones de sus instituciones como en lo que respecta a las características de sus estudiantes. Con ello, en lugar de orientarse principalmente a la formación de estudiantes provenientes desde las clases medias y altas mediante la transferencia de una "alta cultura", los sistemas universitarios contemporáneos se rigen por un principio de diversidad en su forma de gobierno, funciones y estudiantes. Este cambio cultural provoca, a su vez, un mayor interés de estudiantes, familias, empresas y gobiernos en las características de la enseñanza impartida en las universidades impulsando, directa e indirectamente, la necesidad de crear indicadores objetivos de las instituciones de educación superior (van Vught, 2009).

La relevancia de estos mecanismos de aseguramiento de la calidad ha motivado a distintos observadores a desarrollar teorías sociológicas con distintos 
niveles de abstracción para explicar su adopción en los sistemas de educación contemporáneos. En esta dirección, la explicación dominante sugiere que estos mecanismos emergen como resultado de la creciente colonización de la vida académica por parte de las lógicas económicas de oferta y demanda (Slaughter y Leslie, 2001; Slaughter y Rhoades, 2004). Dicho análisis enfatiza que las nuevas técnicas de rendición de cuentas representan una tecnología política a través de la cual se busca imponer una racionalidad económica en ámbitos tradicionalmente ajenos a esta lógica, como es el caso de las universidades (Shore y Wright, 2000; Olssen y Peters, 2005; Deem y Brehony, 2005). Desde este punto de vista, la introducción de mecanismos de aseguramiento de la calidad es un intento de subordinar las actividades de las universidades a las necesidades del mercado laboral, con el resultado de convertir a las instituciones de educación superior (IES) en organizaciones cuyas actividades no difieren respecto de las de las empresas.

Una segunda forma de interpretación sociológica de estos cambios en los sistemas de educación superior comprende la emergencia de estos sistemas como una manera efectiva de asegurar la calidad de las IES. Esta explicación es especialmente preponderante en el discurso de la política pública (para el caso chileno, véase CNA, 2010). Desde esta perspectiva se argumenta que, a través de la evaluación externa de las IES por órganos autónomos, se hace posible resguardar la calidad de la formación universitaria en un contexto de creciente diversidad de funciones, además de elevar la racionalidad en la toma de decisiones de política pública y contribuir a una elección informada por parte de estudiantes, empleadores y familias.

En contraste, los estudios sociohistóricos acerca de la evolución de las formas de confianza en las instituciones parecen ser todavía una excepción en el campo de la educación superior (véase Scott, Gallacher, y Parry, 2017). A nuestro juicio, esto tiene importantes consecuencias negativas para la reflexión acerca de la evolución de las universidades, en tanto se pierde la posibilidad de comprender el impacto de la imposición de los sistemas de aseguramiento de la calidad desde una perspectiva histórica. Quizá más importante, derivado de lo anterior, se naturaliza la organización de los sistemas actuales de aseguramiento de la calidad como la única alternativa para generar confianza en las universidades.

\section{La teoría de sistemas sociales y el análisis de la confianza en las organizaciones}

La teoría de sistemas sociales desarrollada por Luhmann ofrece un punto de partida interesante para realizar un análisis histórico de la evolución de los mecanismos de aseguramiento de la calidad guiado por hipótesis sociológicas estructurales. Como mencionamos, el fenómeno de la confianza es fundamental en este respecto, en tanto toda forma de evaluación de las IES presupone confianza en su capacidad de representar correctamente sus actividades frente a otros observadores.

Para la perspectiva sistémica se parte de la premisa que la confianza es un fenómeno eminentemente paradojal (Luhmann, 1996). Por una parte, su emergencia es improbable, pues no asegura que las expectativas de los involucrados se cumplan satisfactoriamente. Por otra, a pesar de esta incertidumbre, la confianza es necesaria para lidiar con las complejidades de la sociedad contemporánea. Esta paradoja se expresa de manera especialmente marcada en el caso de las organizaciones. Nada asegura que los sistemas organizacionales desempeñen adecuadamente sus actividades, pero aun así se espera que ellos puedan cumplir las expectativas que su misma existencia hace posible establecer.

La teoría de sistemas pone el foco, en consecuencia, en observar el problema de la confianza como un problema comunicacional y no fenomenológico (Morgner, 2018). Desde esta perspectiva, el problema de la confianza/desconfianza se entiende funcionalmente y se relaciona con el nivel operativo 
de los sistemas para comunicar las decisiones como solución/problema (Luhmann, 1996). En este sentido, la confianza deja de ser interpretada como un evento meramente subjetivo y se transforma en un proceso operado por el propio sistema, entre quienes participan en la comunicación. Participar en una relación de confianza implica así probabilizar que la oferta que se da a conocer es comunicativamente pertinente para aceptarla y útil para la creación de expectativas con base en ella (Renn y Levine, 1991; Javala, 2006).

Formulado de esta manera, la forma dominante de confianza no depende de sus características intrínsecas sino de la evolución sociohistórica que favorece ciertas modalidades de relaciones sociales por sobre otras. En esta dirección, las formas de confianza de la sociedad moderna presentan atributos históricos específicos que la diferencian respecto de las formas de confianza de sus formaciones sociales precedentes, ya que el riesgo es lo que se activa antes de entrar en una relación. Contrario de Aristóteles, en quien la confianza era interpretada desde un horizonte teleológico, en la que el ser humano aspiraba a su propia perfección, movido por su naturaleza y actuando también por el bien del otro para mantener el interés de la polis, la cual era la más perfecta y hermosa (Luhmann, 2007), hoy en día la relevancia de la confianza no depende ya de su cercanía a un orden natural definido de antemano sino más bien de qué tanto ella permite probabilizar que las partes involucradas solucionen problemas de incertidumbre mediante mecanismos cada vez más formales, racionales y, sobre todo, de orden impersonal (Lyotard, 1984; Esposito, 1997; Cohen, 1999; Meyer et al., 1997).

Eventos como el debilitamiento del rol del Estado benefactor (Offe, 1999), el incremento de la preocupación por la individualización (Beck y Gernsheim, 2002; Giddens, 1996) y la expansión de derechos civiles, políticos y sociales (Mitzal, 1996), entre otros, apuntan en este sentido. Esto ha resultado en la emergencia de nuevas formas de confianza institucional a través de organizaciones dedicadas a la gestión y manejo del conocimiento (Nowotny, 2003; Grundmann, 2017). Dentro de este contexto no debe sorprender, por lo tanto, que su influencia haya aumentado de manera notable en las últimas décadas, con la emergencia de una clase gerencial, capaz de convertir aquello que se percibe como desconocido, peligroso o conflictivo en materias acerca de las cuales decidir racionalmente por medio de la aplicación de su conocimiento experto (Cetina, 1997; Maasen y Weingart, 2011). El objetivo del conocimiento deja de ser visto como la búsqueda de una verdad natural o la especialización en un ámbito particular de experticia y se transforma, en cambio, en la gestión de incertidumbre a través de las oportunidades de evaluación e intervención que éste ofrece (Collins y Evans, 2002; Nowotny, 2003).

De esta manera, la confianza en la experticia se fundamenta actualmente en la confianza de que este conocimiento es útil para la construcción de creencias firmes, adecuadas para resolver problemas prácticos y no por ser este saber cercano a una verdad definida filosóficamente (Mariñez, Labraña y Matus, 2019). Como resume Nahessi (2004), esta forma de confianza depende, antes que nada, de que la experticia se ajuste a los contextos y tenga en cuenta para quién y qué problemas puede ella resolver.

\section{Cambios en las formas de confianza y la emergencia de los sistemas de aseguramiento de la calidad: una hipótesis interpretativa desde la teoría de sistemas sociales}

Siguiendo lo anterior, es necesario hacerse cargo de la historicidad señalada de la confianza en los sistemas de aseguramiento de la calidad al examinar su función en la sociedad. Como otras organizaciones basadas en la experticia, estos sistemas se hacen cargo de la resolución de un problema particular a través de la toma de decisiones basadas en su juicio 
experto (Javala, 2006). En el caso de las agencias encargadas del aseguramiento de la calidad de la educación superior, ellas buscan resolver el problema de cómo crear confianza en los productos de determinadas instituciones mediante la toma de decisiones fundadas técnicamente para, mediante la comunicación de dichas decisiones, persuadir a los distintos actores sociales interesados de la conveniencia de aceptar el riesgo de confiar en ellas.

Según el diagnóstico de Luhmann (1996, 2000) respecto a los cambios en las formas dominantes de confianza, las nuevas formas de conectar expectativas dependen de una confianza mucho más tecnificada frente a la diversidad de formas de conocimiento que coexisten en el mundo. Los recientes mecanismos de confianza dependen de un conocimiento experto cuyo rol es gestionar dicha incertidumbre en un contexto en que ni la iglesia, la política o el prestigio académico pueden ya lidiar, como antes, con la complejidad de la sociedad contemporánea (Barnett, 2000). A nuestro juicio, las razones estructurales de esta transformación en la existencia de una sociedad más diferenciada, en la que lo familiar en las personas se fragmenta, porque se confía menos en el aseguramiento de la validez de los enunciados por su origen religioso o prestigio institucional. Dicho de otra forma, la familiaridad de las sociedades tradicionales se fragmenta cuando prima una confianza tecnificada y diversa institucionalmente, cuya relevancia depende de su habilidad de resolver problemas específicos y no del hecho de compartir creencias idénticas sobre el funcionamiento correcto de la sociedad (Habermas, 1990).

En este nuevo contexto, las relaciones interpersonales deben orientarse hacia mecanismos mucho más formales para estabilizar la confianza de cualquier individuo en las organizaciones. Como explica Cohen (1999: 221), "la confianza interpersonal, como una relación de cara a cara, no puede ser vista como un fenómeno impersonal que se generaliza ya que simplemente no puede ser transferido a otros contextos". Si bien la confianza es improbable en un mundo donde la decepción es una posibilidad presente (Mariñez, 2013), los sistemas expertos son necesarios en la sociedad en tanto permiten generar las necesarias condiciones mínimas para que los individuos puedan regular sus expectativas respecto al operar de las instituciones (Seligman, 1997). Si ellas cumplen o no dichas expectativas depende de cada relación; lo relevante de la existencia de una confianza tecnificada es que ella, como tal, permite establecer de antemano qué es lo posible en relación con cada institución y, de esta manera, contribuir a reducir la incertidumbre con la cual cada individuo debe hoy necesariamente lidiar (Mariñez, Labraña y Matus, 2019).

Como consecuencia, lo que se hace familiar en la sociedad contemporánea es el riesgo manejado mediante confianza técnica. Con ello, la forma dominante de confianza posee una relación mucho más pragmática y funcional $-\mathrm{y}$, a su vez, mucho más multidimensional y reflexiva - para enfrentar la complejidad del mundo, sin poder ya reducirse a un apriorismo solidario moral propio de formaciones sociales precedentes (Durkheim, 1987). En otras palabras, si hoy se requieren mecanismos institucionalizados para absorber la incertidumbre al entrar al "juego de la confianza", es precisamente porque ellos permiten aceptar este riesgo mediante la definición de qué puede esperarse (Faulkner, 2011).

Este cambio implica una transformación en los mecanismos de evaluación de las universidades. La confianza técnica de las sociedades modernas es, de esta forma, distinta de la familiaridad basada en la posición evaluadora privilegiada de la iglesia católica o el conocimiento de la historia de las respectivas instituciones. Ello crea, por lo tanto, la necesidad de confiar en sistemas expertos de los que se presupone la capacidad de gestionar la incertidumbre del mundo a través de su conocimiento especializado. Si bien estos sistemas no pueden predecir que las expectativas depositadas en una institución se 
cumplan, sí permiten convertir un futuro desconocido en un presente posible de controlar para los actores interesados.

Sin embargo, esta opción por el conocimiento experto no siempre fue vista como la opción más confiable para observar las actividades de las instituciones de educación superior. En sus orígenes medievales, como comunidades de maestros y alumnos, las actividades de las universidades eran reguladas por representantes de la iglesia católica, en particular en lo que respecta a las materias que los académicos podían enseñar y los reglamentos a los que debían sujetarse los estudiantes (Nordi, 1992; Dmitrishin, 2013). Como era característico de las sociedades tradicionales, la confianza operaba de forma intuitiva con base en una comprensión casi natural del mundo en que no se cuestionaba la naturaleza divina del orden social, representado por la Iglesia, órgano que determinaba así qué esperar de la educación (Luhmann, 2002).

A medida que la idea de un orden natural fue perdiendo plausibilidad, ella fue reemplazada por una reflexión que enfatiza la contribución nacional de las universidades, sea mediante la formación de profesionales responsables o de las burocracias estatales, la integración de la ciencia con la docencia o por su compromiso político asociado a los ideales de la modernidad (Lyotard, 1984; Labraña, 2018; Mariñez, Labraña y Matus, 2019). En este nuevo contexto, la universidad del Estado pasó a concebirse como representante del ideal de calidad en la educación superior. Mediante su comparación se evaluaba si el resto de las instituciones del sistema poseían una orientación nacional en sus actividades. Así, con la política separada de la iglesia y la moral separada del deber de la obediencia (Koselleck, 2012), este tránsito implicó que la sociedad dejó de coordinar sus expectativas con base en una autoridad religiosa, encargada de dirigir a los seres humanos a su salvación, para enfocarse en adelante en la idea de desarrollo nacional (Foucault, 1995).
Una vez que el vínculo entre ideas nacionales y proyectos de modernización dejó de ser central y el conocimiento científico empezó a ser valorizado por su aplicación práctica, los sistemas de aseguramiento de la calidad se hicieron más dependientes del conocimiento experto. Como hemos mencionado, la forma dominante de confianza en la sociedad contemporánea no se basa ya en ideas de verdad revelada ni en una comprensión histórica de la relevancia de las actividades de las instituciones asociada a un proyecto de desarrollo nacional. En su lugar, la confianza es técnica, lo que significa que ella se sustenta en las capacidades o habilidades de los expertos para solucionar problemas que no se saben manejar (Mariñez, Labraña y Matus, 2019). Los sistemas expertos asumen esta importancia creciente, especialmente en el campo de la educación superior, debido a que se acepta el riesgo de creer que ellos saben cosas que nosotros no. Pero esta necesidad abre paso, paradójicamente, a la sospecha sobre lo presentado, haciendo ver que la relevancia de sus evaluaciones no es tan evidente como podía serlo antes (Luhmann, 2010).

En la siguiente sección se examinará cómo esta transformación en los medios para asegurar la calidad de las instituciones de educación superior puede también rastrearse en el contexto chileno. Si bien existen importantes particularidades en la trayectoria histórica de los sistemas de aseguramiento de la calidad en este país, como la importancia inicial de la iglesia católica y la centralidad hasta mediados de la década de los cincuenta de la universidad estatal y de las instituciones creadas antes de 1981 y hasta mediados de los 2000, estos atributos no contradicen el modelo de desarrollo aquí delineado, sino que más bien demuestran la relevancia de factores nacionales al momento de observar estas transformaciones.

\section{La tecnificación del aseguramiento de la calidad de la educación superior en Chile}

Si bien la emergencia de mecanismos expertos de aseguramiento de la calidad es un fenómeno relativamente 
reciente en la educación superior de América Latina (Brunner, 2014), ella ha impactado fuertemente en la organización de la educación superior en este país. Hasta mediados de la década de los ochenta, las instituciones públicas ubicadas en esta región recibían recursos no sujetos a rendición de cuentas por parte del Estado, en un contexto de alta autonomía académica y fuerte dependencia de recursos fiscales (Levy, 1986). Sólo recientemente, de la mano del aumento de la importancia de instituciones privadas y el retroceso del Estado en la coordinación de sistemas universitarios nacionales (Labraña, 2016), han comenzado a emerger sistemas expertos encargados de evaluar la formación otorgada en las universidades de los sistemas educativos terciarios de la región.

Chile representa un caso notable de este cambio. Por una parte, este sistema ha experimentado transformaciones notables en las últimas décadas. Si bien históricamente su sistema universitario presentó un marcado dominio del Estado en el financiamiento del sector, en que el gobierno optó por financiar de manera similar a instituciones de educación superior privadas y estatales (Levy, 1986). Hoy en día este sistema se ha convertido en un símbolo de los efectos de las reformas de privatización en términos de mercadización, comercialización y mercantilización (Brunner, 2015, 2017; Mariñez, Labraña y Matus, 2019).

Como se verá a continuación, la evolución de los distintos mecanismos utilizados socialmente para asegurar la calidad de la educación superior chilena ha experimentado una evolución similar, pasando por distintos referentes religiosos y políticos, los cuales sólo en las últimas décadas han sido reemplazados por una confianza generalizada en los organismos de evaluación experta. En este sentido, la primera universidad de la Corona creada en el territorio chileno fue la Real Universidad de San Felipe. Fundada en 1738, esta institución de educación superior comenzó a operar recién en 1756, enfocándose en la enseñanza religiosa basada en la obra de Santo Tomás, sin poder alcanzar una existencia autosuficiente en las décadas siguientes. En efecto, a pesar de ser fundada en el periodo colonial tardío, la Real Universidad de San Felipe mantuvo una orientación marcadamente religiosa, en tanto en su organización no penetraron las reformas académicas de raíz moderna del Despotismo Ilustrado Español (Serrano, 1994).

Este carácter religioso de la universidad se expresó en el currículo y métodos de enseñanza de la universidad, fuertemente influidos por la doctrina escolástica (Serrano, 1994). De esta manera, la iglesia católica se constituyó como la garante de la calidad de las actividades de esta institución. Así, obtener un grado académico en este periodo requería jurar obediencia a la iglesia, además del compromiso del egresado de ser fiel a los dogmas e ideas cristianas y respetar las autoridades religiosas (Jobet, 1970).

Una vez concluido el proceso de independencia nacional a inicios del siglo XIX, los intelectuales chilenos de este periodo tendieron a adoptar una valoración crítica de la que fuese la Universidad de San Felipe. En su reemplazo, se fundó en 1842 la Universidad de Chile mediante la fusión de distintos establecimientos educacionales de la Colonia, incluyendo la Universidad Real, el Instituto Carolino y la Academia de San Luis. Como quedó expresado en el discurso inaugural de su primer rector, el venezolano Andrés Bello (1997), su misión fue descrita como contribuir al progreso nacional, ayudando a resolver las necesidades del país y formando sus nuevas élites culturales y profesionales (Labraña, 2018).

La cuestión del aseguramiento de la calidad de la educación superior apareció nuevamente con la creación de alternativas no estatales de formación terciaria. En 1888, monseñor Mariano Casanova, arzobispo de Santiago, fundó la Universidad Católica en respuesta a las tendencias a favor de la secularización percibidas en los miembros de la Universidad de Chile. Esta institución, junto con las universidades privadas creadas durante las décadas siguientes - la Universidad de Concepción (1919), la Universidad Técnica Federico Santa María (1926), la 
Universidad Católica de Valparaíso (1928), la Universidad Austral de Chile (1954) y la Universidad del Norte (1956) - , fueron obligadas a hacer rendir a sus estudiantes exámenes de finales de curso ante comisiones académicas de la universidad estatal.

Esta regulación del sistema universitario chileno a cargo de la estatal Universidad de Chile quedó registrada en distintos cuerpos legales. El Decreto con Fuerza de Ley 7500 (Ministerio de Educación Pública, 1927) reconoció la autonomía administrativa de las universidades y su capacidad de definir "todo lo inherente a su organización, ubicación y funcionamiento" (artículo 26). Sin embargo, al mismo tiempo, esta normativa estableció que la entrega de grados académicos y títulos profesionales por parte de las universidades privadas debía estar subordinada al control de sus actividades académicas por parte de la Universidad de Chile, en directa representación del gobierno. En breve, entonces, la cuestión de la calidad de las actividades de las universidades fue resuelta a partir de la acción de un organismo estatal (la Universidad de Chile) encargado de evaluar sus actividades y decidir sobre su adecuado funcionamiento.

En la misma dirección, el Decreto Ley 4807 (Ministerio de Educación Pública, 1929), Estatuto Orgánico de la Enseñanza Universitaria, que habría de reemplazar al Decreto 7 500, determinó que la Universidad de Chile era la institución a cargo de la supervigilancia del conjunto del sistema chileno de la educación superior. El decreto estableció que el Consejo Universitario, compuesto por el rector de la Universidad de Chile, los decanos de las facultades y el secretario general de la Universidad, en conjunto con los consejeros designados por el presidente de la República y representantes de la educación primaria y secundaria (artículo 4), era el encargado, entre otras tareas, de reglamentar las criterios de acceso a las universidades (artículo 14, i) y de proponer al gobierno el número de alumnos que ellas podrían presentar a examen cada año (artículo 14, k).

Además de regular el acceso de los estudiantes a las universidades privadas, esta normativa definió los requisitos de apertura y funcionamiento de las instituciones de educación superior. Todo establecimiento docente que buscase presentar alumnos para rendir exámenes que condujesen a la obtención de los grados y títulos que fuesen otorgados por la Universidad de Chile, debía antes obtener la autorización del gobierno, previo informe de su Consejo Universitario (artículo 77). A su vez, toda enseñanza cuyo fin fuese otorgar grados o títulos conferidos por la Universidad de Chile debía adaptarse a los planes de estudio y programas aprobados por el Consejo Universitario para las escuelas de esta institución nacional (artículo 78).

Este aseguramiento del valor académico y profesional de las credenciales otorgadas por las universidades privadas a partir de la evaluación de la Universidad de Chile se mantuvo en la legislación nacional hasta mediados de la década de los cincuenta. Esto muestra cómo la confianza en el aseguramiento de la calidad de la educación superior se depositaba en la capacidad de evaluación de una élite ilustrada identificada con la realización de objetivos colectivos (Serrano, 1994). Con el apoyo del Estado, la Universidad de Chile se constituyó, así, como la cúspide del sistema universitario chileno, regularizando el valor de las credenciales educacionales otorgadas por las otras instituciones. ${ }^{1}$ Esta confianza en el rol mediador de la Universidad de Chile no se basó, sin embargo, en la formalidad de

\footnotetext{
${ }^{1}$ Este principio tuvo claras limitaciones al aplicarse a la organización del sistema universitario. En la práctica, los egresados de las universidades privadas eran generalmente contratados con independencia de si sus títulos habían sido previamente validados por los académicos de la Universidad de Chile. En respuesta a esta situación, distintas normativas relacionadas a áreas profesionales específicas, establecieron la validez de los conocimientos de egresados de diferentes carreras profesionales, especialmente desde la década de los cincuenta en adelante (Campos, 1960).
} 
sus evaluaciones. Precisamente por su asociación intrínseca con objetivos nacionales, esta universidad era vista como cualitativamente superior al resto de instituciones y, por ello, como la única entidad capaz de evaluar la calidad de sus actividades sin que fuese necesario que ella misma se sujetara al control de otras organizaciones.

Esta asociación entre universidad estatal, objetivos de desarrollo nacional y la capacidad intrínseca de asegurar la calidad del resto del sistema universitario, se hizo inviable a partir de la década de los cincuenta. Por una parte, las nuevas universidades habían aumentado su matrícula ante la mayor demanda profesional del sector laboral, superando la capacidad de respuesta de los académicos de la Universidad de Chile. Por otra parte, la universidad estatal también comenzó durante este periodo a perder su monopolio del conocimiento superior frente a otras instituciones que, como apunta Levy (1986), desarrollaban actividades similares en términos de docencia, investigación y compromiso social.

Para el aseguramiento de la calidad, esto implicó el reemplazo de la regulación a través de la evaluación de la Universidad de Chile por un mecanismo basado en una confianza institucionalizada en el prestigio. Si bien no existe una legislación general formulada durante este periodo, se aceptó en el discurso público que las nuevas universidades debían ser reconocidas por el Estado para poder otorgar títulos profesionales y que, una vez obtenida la autorización, estas instituciones, con independencia de su propietario, podían operar de manera autónoma y recibir recursos públicos no sujetos a rendición de cuentas por el cumplimiento de su inherente función pública (Brunner y Briones, 1992; Bernasconi y Rojas, 2004).

Este arreglo de "autonomía privilegiada" (Brunner y Briones, 1992) se mantuvo hasta la reforma universitaria impuesta durante la dictadura militar del general Pinochet. El Decreto con Fuerza de Ley 1 (1981) estableció que las nuevas universidades tenían que constituirse como personas jurídicas de derecho privado sin fines de lucro (artículo 15). Esta normativa definió que, después de obtener la aprobación de los ministerios de Educación y del Interior, las nuevas IES debían presentar sus programas de estudio a académicos de una universidad creada antes de 1981 o de una de sus sedes derivadas (artículo 24). Así, las cinco primeras promociones de alumnos en carreras que finalizasen en la obtención de un grado académico debían rendir sus exámenes finales ante comisiones mixtas, conformadas por profesores de la nueva universidad y de una universidad tradicional, siendo decisiva la opinión de los académicos de esta última en el caso de divergencias frente a su evaluación (artículo 26).

La confianza en las universidades tradicionales como garantes de la calidad del sistema se mantuvo hasta finales de la dictadura militar, siendo reconocida en el discurso oficial su superioridad intrínseca a otras instituciones. Como argumentó el ministro de Educación de la época, Gonzalo Vial (1979), ellas representaban el estándar de calidad al cual necesariamente tenían que aspirar las nuevas universidades privadas. De esta manera, el mecanismo de examinación de las nuevas universidades a cargo de las universidades tradicionales permaneció siendo altamente influyente durante este periodo, basándose en su prestigio en el discurso, sin ser necesario que esas instituciones fuesen a su vez evaluadas externamente.

Este mecanismo de regulación de la calidad entró en crisis con la proliferación de nuevas universidades privadas a finales de la década de los ochenta. Entre 1988 y 1990 se autorizó el funcionamiento de 25 universidades, cuyas demandas por la evaluación de sus programas sobrepasaron las capacidades académicas de las instituciones tradicionales (Bernasconi y Rojas, 2004). Ello implicó la necesidad de pasar de una confianza basada en el prestigio histórico de las instituciones a una confianza más tecnificada, concebida como resultante de una medición basada en los criterios de órganos expertos. 
Pocos días antes del término de la dictadura militar se promulgó la Ley 18 962, Orgánica Constitucional de Enseñanza (Ministerio de Educación, 1990). Esta normativa creó un nuevo mecanismo de licenciamiento (Consejo Superior de Educación, CSE), al cual se le asignó la responsabilidad de evaluar los proyectos institucionales de las nuevas universidades que aspirasen a tener reconocimiento oficial y de verificar y evaluar su correcto desarrollo por un periodo de entre seis hasta un máximo de 11 años.

La evaluación del CSE estuvo estructurada en las siguientes fases. En primer lugar, se analizaba el proyecto institucional presentado por la universidad, así como sus carreras y programas conducentes a títulos y grados, considerando aspectos docentes, didácticos y técnico-pedagógicos de la institución en formación; sus planes y programas de estudios; sus recursos físicos y de infraestructura y, finalmente, su disponibilidad de recursos económicos y financieros. A continuación, el Consejo debía evaluar anualmente el avance del proyecto institucional, formulado sobre la base del análisis de las variables mencionadas, así como de los resultados de la examinación de cursos de la universidad a cargo de pares expertos definidos por el CSE. Finalmente, de acuerdo con esta evaluación, el Consejo debía decidir si acaso debía otorgar o no autonomía a las universidades privadas (CSE, 1991).

El CSE continuó operando casi sin cambios en las siguientes décadas. Sin embargo, sus limitaciones comenzaron rápidamente a hacerse visibles. Las nuevas universidades privadas, una vez obtenida su autonomía, así como también las universidades estatales y las instituciones derivadas de las universidades privadas creadas antes de 1981, tendían a abrir nuevas sedes a lo largo del país, impulsadas por la necesidad de atraer mayores recursos de los estudiantes y sus familias en un entorno económicamente competitivo, sin prestar atención a sus fines originales. De 60 universidades con 70 sedes en 1990 se pasó a 68 con 103 sedes en 1997 y, luego, a 62 con un total de 116 sedes en 2002 (Zapata et al., 2003).
Con el objetivo de resolver este problema, en 1999 se creó la Comisión Nacional de Acreditación de Pregrado. Este órgano estuvo destinado a iniciar procesos piloto de acreditación, de carácter voluntario, a instituciones autónomas de educación superior. A pesar de que sus evaluaciones no fueron establecidas como condición para acceder a recursos fiscales, sí fueron valoradas por las universidades como un medio de asegurar su prestigio institucional frente a otros posibles competidores (Salazar, 2013).

Con base en la experiencia de esta comisión, en 2006 se creó la Comisión Nacional de Acreditación (CNA), con el objetivo de dar garantía pública de la calidad de las IES después de que les fuese otorgada la autonomía por parte del CSE. Como apuntaba el mensaje que acompañó al proyecto de ley en cuestión, la evaluación de este organismo haría posible que "las decisiones que los ciudadanos adopten con relación a los servicios proporcionados por el sistema de educación superior" estuviesen "apoyadas por antecedentes sólidos, provenientes de la propia comunidad técnica, profesional y académica, y refrendados y sustentados por el Estado" y no en el mero prestigio de las universidades (Ministerio de Educación, 2006: 6).

En la actualidad, este órgano tiene las funciones de administrar la acreditación de instituciones autónomas de educación superior; pronunciarse sobre las solicitudes de autorización de las agencias encargadas de la acreditación de carreras y programas de pregrado, de magíster y programas de especialidad en el área de salud y mantener sistemas de información para los actores interesados. Para cumplir con este cometido, la CNA debe resguardar la calidad de las IES con base en dos procedimientos claramente diferenciados. Por una parte, mediante la acreditación institucional, encargada de certificar el cumplimiento del proyecto de la institución y la existencia dentro de mecanismos eficaces de autorregulación y aseguramiento de la calidad. Por otra parte, mediante la acreditación de carreras y programas a cargo de 
agencias privadas, proceso mediante el que se certifica su calidad en función de los propósitos declarados por cada entidad y los criterios establecidos por las comunidades académicas y profesionales expertas. Este proceso es realizado por agencias nacionales o internacionales que, como condición de operación, deben poseer la autorización de la CNA y seguir sus criterios de evaluación.

Esta nueva importancia del conocimiento experto en la evaluación de las universidades ha afectado fuertemente cómo el Estado distribuye sus recursos en el sector de la educación superior, pasando de criterios históricos, centrados en el prestigio de las instituciones, a criterios técnicos, centrados en sus resultados en la CNA (Labraña y Rodríguez, 2017). Por su parte, si bien no existen estudios en ámbitos que permitan hacer una comparación histórica de cómo estas transformaciones en los mecanismos de aseguramiento de la calidad de la educación superior han afectado las expectativas de estudiantes y representantes del mundo laboral, la literatura disponible sugiere que la institucionalización del conocimiento experto en este campo ha resultado en la disminución de la relevancia de métodos alternativos de evaluación de las universidades (Torres Rojas, 2012; IPSOS, 2010).

Como puede apreciarse a partir de este recorrido de los mecanismos utilizados en la historia de la educación chilena para asegurar la calidad de las universidades, la tecnificación de su evaluación es históricamente nueva, si bien se corresponde con las tendencias globales delineadas en la sección anterior. La evaluación de las instituciones de educación superior no sigue una correspondencia con una verdad revelada por la iglesia católica, la capacidad de alinearse con objetivos nacionales o el prestigio histórico de las universidades tradicionales. En cambio, la confianza está puesta ahora en la capacidad de sistemas expertos de evaluar la calidad de las universidades y, de esta manera, asegurar la generación de información válida sobre sus actividades académicas.
No debe sorprender, en este contexto, que las críticas al sistema de acreditación de las universidades chilenas en el marco del debate actual sobre la reforma de la educación no aspiren a retornar a uno de los métodos anteriores de aseguramiento de la calidad, sino más bien a cambiar los criterios de evaluación, siempre bajo el presupuesto que el conocimiento experto es la alternativa más confiable para este propósito (González y Espinoza, 2017).

\section{Conclusiones}

Como se ha argumentado, el conocimiento experto es clave para la producción de confianza en la sociedad contemporánea. A pesar de esta centralidad, no parece haberse analizado de manera suficiente su historicidad ni su relación con la instalación de mecanismos de rendición de cuentas, especialmente en la educación superior chilena. En efecto, si las oportunidades que pueden desprenderse del conocimiento acerca de las instituciones de educación superior dependen en gran medida de los expertos que los evalúan, entonces su emergencia e institucionalización no pueden comprenderse sólo como un procedimiento científico-técnico, sino deben considerarse como resultado del grado en que las formas de evaluación se ven como confiables (Wynne, 1992; Collins y Evans, 2002).

En particular, en este artículo se ha sostenido que la confianza es un mecanismo que tiene como función principal permitir enfrentar problemas de incertidumbre y que sus formas dominantes varían históricamente. Como se ha examinado en este artículo en relación con la evaluación de la calidad de la educación superior, si con referencia al saber de la iglesia, la orientación nacional de los saberes o el prestigio histórico se podía confiar en la calidad de las universidades con base en su familiaridad y la presunción de su carácter cuasi-natural, la tecnificación de la evaluación abre también la posibilidad de desconfiar. La confianza ahora no es sólo necesaria sino también riesgosa, pues ella se comunica como 
decisión y, con ello, puede también cuestionarse en función de las características de su autor.

La educación superior chilena aparece como un campo en que estos mecanismos han adquirido especial relevancia en las últimas décadas. Ella se inició ya en el siglo XIX, con la creación de la Universidad de San Felipe y la preocupación acerca del carácter religioso de su enseñanza. A continuación, la Universidad de Chile fue concebida como la institución responsable de controlar la calidad de las restantes instituciones educacionales. Este rol coordinador de la universidad estatal entró en crisis a partir de la década de los cincuenta y se reemplazó por una confianza basada en la similitud de las actividades de las instituciones. Después de la apertura de nuevas universidades privadas tras la reforma de 1981, esta similitud perdió su anterior carácter autoevidente y la regulación del sistema se asignó al Ministerio de Educación y a las universidades creadas antes de 1981, concebidas como capaces de evaluar a las restantes instituciones debido a su prestigio histórico, y al Ministerio del Interior. Con el retorno a la democracia en 1990, las "universidades tradicionales" perdieron relevancia ante la acción de organismos como el CNED y la CNA, que hoy deben evaluar distintas instituciones en función de criterios técnicos.

Este análisis permite reconocer la dependencia histórica de las variaciones en la confianza. Hoy resulta tan inimaginable la evaluación de las universidades chilenas de acuerdo con los criterios de la iglesia católica, como habría sido en ese periodo relegar su coordinación a sistemas expertos. En este sentido, la confianza en las formas de evaluación es histórica y, con ello, dependiente de la estructura de la sociedad y no de atributos particulares de este proceso. La pérdida de plausibilidad de explicaciones naturales y el aumento de incertidumbre de la sociedad contemporánea son variables centrales en este respecto.

Futuros estudios pueden seguir esta línea de desarrollo y analizar cómo estos sistemas de aseguramiento de la calidad han sido observados socialmente. Siguiendo la distinción propuesta por Luhmann (1975) entre interacción, organización y sociedad, el análisis desarrollado en este artículo se ha enfocado en cómo cambios sociales han configurado la plausibilidad de confiar en determinadas organizaciones. Ello no asegura, sin embargo, que sus decisiones sean integradas en el plano de la interacción. Por el contrario, si se siguen investigaciones en el área (Torres Rojas, 2012), se puede hipotetizar una creciente diversificación de los modos de apropiación de las decisiones técnicas en las instituciones de educación superior. Futuras investigaciones podrían complementar este análisis comparándolo con la forma en que los mecanismos de aseguramiento de la calidad fueron apropiados por distintos actores durante las décadas anteriores.

En lo que respecta a la relevancia de estos desarrollos para la formulación de políticas públicas, en opinión de los autores es un error restringir la relevancia de estos resultados al campo de los estudios históricos en educación superior. Los encargados de la formulación de políticas públicas bien podrían utilizar estos hallazgos para observar los condicionantes de su confianza en las decisiones de organismos técnicos. Esto podría, a su vez, resultar en un análisis más preciso de las posibilidades de estos mecanismos y el reconocimiento de la importancia de la confianza por parte de otros actores. El conocimiento experto no es suficiente por sí solo, sino que, como se argumentó en este artículo, requiere de confianza en su validez. Para el caso chileno, este es una materia central para la política educativa. 


\section{Referencias}

Barnett, R. (2000), Realizing the university in an age of supercomplexity, Buckingham, The Society for Research into Higher Education \& Open University Press.

Beck, U. y E. Gernsheim (2002), La individualización. El individualismo institucionalizado y sus consecuencias sociales y políticas, Barcelona, Paidós.

Bello, A. (1997), "Address Delivered at the Inaguration of the University of Chile (1843)", en A. Bello (ed.), Selected writings of Andres Bello: translated from the Spanish by Frances M. López-Morillas, Nueva York/Oxford, Oxford University Press, pp. 124-137.

Bernasconi, A. y F. Rojas (2004), Informe sobre la educación superior en Chile: 1980-2003, Santiago, Chile, Editorial Universitaria.

Brunner, J. J. (2017), "La economía política del capitalismo académico: el caso de Chile", en H. Lavados y A. L. Durán (Eds.), Desafios para el desarrollo universitario en Chile, Madrid, Tirant humanidades, pp. 159-231.

Brunner, J. J. (2015), "Medio siglo de transformaciones de la educación superior chilena: Un estado del arte", en A. Bernasconi (ed.), La educación superior de Chile: transformación, desarrollo y crisis, Santiago, Chile, Ediciones Universidad Católica de Chile, pp. 21-108.

Brunner, J. J. (2014), “La idea de la universidad pública en América Latina: narraciones en escenarios divergentes", Educación XXI, vol. 17, núm. 2, pp. 17-34.

Brunner, J. J. y G. Briones (1992), "Higher education in Chile: effects of the 1980 Reform", en L. Wolff y D. Albrecht (eds.), Higher education reform in Chile, Brazil and Venezuela, Washington, The World Bank (World Bank Paper LATHR No 34 ).

Campos, F. (1960), Desarrollo educacional 1810-1960, Santiago, Chile, Editorial Andrés Bello.

Cetina, K. K. (1997), "Sociality with objects: social relations in postsocial knowledge societies", Theory, Culture E̊ Society, vol. 14, núm. 4, pp. 1-30.

Cohen, J. (1999), "Trust, voluntary association and workable democracy", en M. Warren (ed.), Trust and democracy, Cambridge, Cambridge University Press.
Collins, H. y R. Evans (2002), "The third wave of science. Studies of experience and expertise", Social Studies of Science, vol. 32, núm. 2, pp. 235-296.

Comisión Nacional de Acreditación (GNA) (2010), Aseguramiento de calidad en la educación superior: Cuatro años de CNA-Chile. Memoria Institucional 2007-2010, Santiago, Ediciones CNA-Chile.

Consejo Superior de Educación (1991), Un año de trabajo, Santiago, Chile, Consejo Superior de Educación.

Decreto con Fuerza de Ley 1 (1981), Fija normas sobre universidades, Santiago, Chile, Ministerio de Educación Pública.

Deem, R. y K. J. Brehony (2005), "Management as ideology: the case of 'new managerialism'in higher education", Oxford Review of Education, vol. 31, núm. 2, pp. 217-235.

Dmitrishin, A. (2013), "Deconstructing distinctions. The European University in comparative historical perspective", Entremons. UPF Fournal of World History, núm. 5, pp. 1-18.

Durkheim, E. (1987), La división del trabajo social, Barcelona, Akal.

Esposito, E. (1997), "Fikton und Virtualität", en S. Krämer (ed.), Medien computer realität: wirklichkeitsvorstellungen und neue medien, Frankfurt am Main, Suhrkamp Verlag, pp. 269-296.

Faulkner, P. (2011), Knowledge on trust, Oxford, Oxford University Press.

Foucault, M. (1995), Tecnologías del yo y otros textos afines, Madrid, Paidós.

Giddens, A. (1996), "Modernidad y autoidentidad. Las consecuencias perversas de la modernidad", en J. Beriain (ed.), Modernidad, contingencia y riesgo, México, Anthropos, pp. 33-72.

González, L. E. y O. Espinoza (2017), "Educación superior bajo el segundo gobierno de Michelle Bachelet: una mirada evaluativa", en Bachelet II: El dificil camino hacia un Estado democrático social de derechos, Santiago, Chile, Barómetro de Política y Equidad, pp. 167-184. 
Grundmann, R. (2017), "The problem of expertise in knowledge societies", Minerva, vol. 55, núm. 1, pp. 25-48.

Habermas, J. (1990), Teoría de la Acción Comunicativa. Vol. II: Crítica de la razón funcionalista, México, Taurus.

IPSOS (2010), Informe Final "Estudio exploratorio sobre efectos de la Acreditación Institucional en la calidad de la educación superior en Chile": Preparado para la Comisión Nacional de Acreditación, Santiago, Chile, IPSOS.

Javala, J. (2006), Trust as a decision. The problems and functions of trust in Luhmannian system theory, Helsinki, University of Helsinki.

Jobet,J. C.(1970),Doctrinay praxisdeloseducadoresrepresentativos chilenos, Santiago, Chile, Editorial Andrés Bello.

Kim, J. Y. (2014), Human Capital in the 21st Century, Washington, World Bank.

Koselleck, R. (2012), Historias de conceptos. Estudios sobre la semántica y pragmática del lenguaje político y social, Madrid, Trotta.

Labraña, J. (2018), "De qué hablamos cuando hablamos de universidades. El Estado chileno y la idea contemporánea de la universidad", Avaliação: Revista da Avaliação da Educação Superior, vol. 23, núm. 3, pp. 834-847.

Labraña, J. (2016), "El concepto de sistema de los sistemas de educación superior: el caso chileno", Calidad en la Educación, núm. 44, pp. 276-299.

Labraña, J. y Rodríguez, J. (2017). "Estado y universidad en Chile: problemas de distinción en torno a su función pública", Pensamiento Educativo. Revista De Investigación Educacional Latinoamericana, vol. 54, núm. 1, pp. 1-19.

Lemaitre, M. J. (2004), "Development of external quality assurance schemes: An answer to the challenges of higher education evolution", Quality in Higher Education, vol. 10, núm. 2, pp. 89-99.

Lemaitre, M. J. y M. E. Zenteno (2016), "La calidad de la formación y el valor de la diversidad", en La función formativa de la Educación Superior, Santiago, Chile, Aequalis, Foro de Educación Superior, pp. 11-73.

Levy, D. C. (1986), Higher education and the state in Latin America: private challenges to public dominance, Chicago/ Londres, The University of Chicago Press.
Ley Orgánica Constitucional de Enseñanza (1990), Ley 18 962, Santiago, Chile, Ministerio de Educación.

Luhmann, N. (2007), La Sociedad de la Sociedad, México, Editorial Herder.

Luhmann, N. (2002), Das Erziehungssystem der Gesellschaft, Frankfurt am Main, Suhrkamp.

Luhmann, N. (2000), Organisation und Entscheidung, Opladen/Wiesbaden, Westdeutscher Verlag GmbH.

Luhmann, N. (1996), Confianza, México, Anthropos.

Luhmann, N. (1975), "Interaktion, organisation, gesellschaft", en N. Luhmann (ed.), Soziologische aufklärung 2: Aufsätze zur theorie der gesellschaft, Wiesbaden, VS Verlag für Sozialwissenschaften, pp. 9-24.

Luhmann, N. y K.-E. Schorr (2000), Problems of reflection in the system of education, Münster/Nueva York, Waxmann.

Luke, C. (2006), "Capital and knowledge flows: global higher education markets", Asia Pacific fournal of Education, vol. 25, núm. 2, pp. 159-174.

Luhmann, N. (2010), Organización y decisión, Ciudad de México, Editorial Herder.

Lyotard, J. F. (1984), The postmodern condition: a report on knowledge, Manchester, Manchester University Press.

Maasen, S. y P. Weingart (2011), "What's new in scientific advice to politics", en S. Maasen y P. Weingart, Democratising expertise. Exploring novel forms of scientific advice in political decision-making, Dordrecht, Springer.

Mariñez, C. (2013), "Una mirada sistémica al problema de la confianza en la cultura organizacional", en A. Urquiza, H. Cadenas y M. Arnold (eds.), La organización de las organizaciones sociales: aplicaciones desde perspectivas sistémicas, Santiago, Ril Editores.

Mariñez, C., J. Labraña y T. Matus (2019), “Observando la conversión de la experticia científica en confianza pública desde el modelo sistémico funcional y el modelo pragmático formal", Cinta De Moebio, núm. 65, pp. 209-226.

Meyer, J. W., Boli, J., Thomas, G. M., y Ramirez, F. (1997), "World society and the Nation-State", American fournal of Sociology, vol. 103, núm. 1, pp. 144-181. 
Ministerio de Educación (2006), Historia de Ley $\mathcal{N}^{\circ} 20.129$. Establece un sistema nacional de aseguramiento de la calidad de la educación superior, Santiago, Chile, Ministerio de Educación.

Ministerio de Educación Pública (1990), Ley Orgánica Constitucional de Enseñanza, Núm. 18 962, Santiago, Chile, Ministerio de Educación Pública.

Ministerio de Educación Pública (1929), Estatuto Orgánico de la Enseñanza Universitaria, Santiago, Chile, Ministerio de Educación Pública.

Ministerio de Educación Pública (1927), Reforma Educacional, Decreto con Fuerza de Ley N ${ }^{\circ} 7$ 500, Santiago, Chile, Ministerio de Educación Pública.

Mitzal, B. (1996), Trust in modern societies, UK, Polity Press.

Morgner, C. (2018), "Trust and society: suggestions for further development of Niklas Luhmann's theory of trust", Canadian Sociological Association, vol. 55, núm. 2, pp. 232-255.

Nassehi, A. (2004), "What do we know about knowledge? An essay on the knowledge society", Canadian fournal of Sociology/Cahiers Canadiens De Sociologie, vol. 29, núm. 3, pp. 439-449.

Neave, G. (1998), "The evaluative state reconsidered", European Gournal of Education, vol. 33, núm. 3, pp. 265-284.

Nordi, P. (1992), "Relations with authority", en W. Rüegg (ed.), A history of the universities in Europe (vol. I. Universities in the Middle Ages), Cambridge, Cambridge University Press, pp. 77-107

Nowotny, H. (2003), "Dilema of expertise. Democratising expertise and socially robust knowledge", Science and the Public Policy, vol. 30, núm. 3, pp. 151-156.

Offe, C. (1999), "How can we trust our fellowships?", en M. Warren (ed.), Trust and democracy, Cambridge, Editorial Cambridge University Press.

Olssen, M. y M. A. Peters (2005), "Neoliberalism, higher education and the knowledge economy: from the free market to knowledge capitalism", fournal of Education Policy, vol. 20, núm. 3, pp. 313-345.

Renn, O. y D. Levine (1991), "Credibility and trust in risk communication", en R. E. Kasperson y P. J. Stallen (eds.), Communicating risks to the public, Dordrecht, Springer, pp. 175-217.
Rodríguez-Ponce, E. (2009), "El rol de las universidades en la sociedad del conocimiento y en la era de la globalización: evidencia desde Chile", Interciencia, vol. 34, núm. 11, pp. 822-829.

Salazar, J. M. (2013), "Public policy for higher education in Chile: a case study in quality assurance (19902009)", PhD thesis, Melbourne, Centre for the Study of Higher Education, Graduate School of Education, The University of Melbourne.

Salazar, J., y P. S. Leihy (2013), "El Manual Invisible: Tres décadas de políticas de educación superior en Chile (1980-2010)", Archivos Analíticos de Políticas Educativas, vol. 21, núm. 34, pp. 1-38.

Scott, J., J. Gallacher y G. Parry (2017), "Reflections and conclusions", en P. Scott, J. Gallacher y G. Parry (eds.), New languages and landscapes of higher education, Oxford, Oxford University Press, pp. 256-264.

Seligman, A. (1997), The problem of trust, Princeton, Princeton University Press.

Serrano, S. (1994), Universidad y Nación: Chile en el siglo XIX, Santiago, Chile, Editorial Universitaria.

Shore, C. y S. Wright (2000), "Coercive accountability. Audit cultures: anthropological studies in accountability", Ethics and the academy, 18, pp. 57-89.

Slaughter, S. y L. L. Leslie (2001), "Expanding and elaborating the concept of academic capitalism", Organization, vol. 8, núm. 2, pp. 154-161.

Slaughter, S. y G. Rhoades (2004), Academic capitalism and the new economy: markets, state, and higher education, Baltimore, Johns Hopkins University Press.

Torres Rojas, E. (2012), "Acreditación institucional y la mirada de los actores: un estudio cualitativo en universidades privadas de Santiago", Estudios pedagógicos, vol. 38, núm. 2, pp. 221-242.

Välimaa, J. y D. Hoffman (2008), "Knowledge society discourse and higher education", Higher Education, vol. 56, núm. 3, 265-285.

Van Vught, F. (2009), "Diversity and differentiation in higher education", en F. van Vught (ed.), Higher education dynamics, 28. Mapping the higher education landscape: towards a European classification of higher education (Vol. 28), 
Dordrecht, Springer Netherlands, pp. 1-16.

Vial, G. (1979), "Discurso pronunciado por el Señor Ministro de Educación Pública. Don Gonzalo Vial Correa, con motivo de la Ceremonia Académica conmemorativa de los 25 años del Consejo", en 25 Años: Consejo de Rectores de las Universidades Chilenas, Santiago, Chile, Secretaria General, CRUCH, pp. 19-34.

Vidovich, L. y J. Currie (2011), "Governance and trust in higher education", Studies in Higher Education, vol. 36, núm. 1, pp. 43-56.
Wynne, B. (1992). "Misunderstood misunderstanding: social identities and public uptake of science", Public understanding of science, vol. 1, núm. 3, 281-304.

World Bank (2002), Constructing knowledge societies: new challenges for tertiary education. Directions in development, Washington, World Bank.

Zapata, G., F. L. Rojas y N. Fleet (2003), Evolución de las sedes de instituciones de educación superior en Chile. Documento de trabajo. Comisión Nacional de Acreditación de Pregrado, Santiago, Chile, Ministerio de Educación.

\section{Cómo citar este artículo:}

Labraña, Julio y César Mariñez(2021), “¿En qué confiamos al evaluar la calidad de las universidades? Evolución de los mecanismos externos de aseguramiento de la calidad en Chile", Revista Iberoamericana de Educación Superior (RIES), vol. XII, núm. 33, pp. 120-137, DOI: https://doi.org/10.22201/iisue.20072872e.2021.33.861 [Consulta: fecha de última consulta]. 